\title{
Enfrentamiento de la hiponatremia: Más allá de la corrección del sodio. A propósito de un caso clínico
}

\author{
ANDRÉS AIZMAN ${ }^{1}$, SOLEDAD LARRAÍN ${ }^{a}$, LUIS ROJAS ${ }^{1}$
}

'Departamento de Medicina Interna. Facultad de Medicina. Pontificia Universidad Católica de Chile.

anterna. Escuela de Medicina. Pontificia Universidad Católica de Chile.

Recibido el 30 de octubre de 2009, aceptado el 25 de agosto de 2010.

Correspondencia a: Dr. Andrés Aizman Sevilla Marcoleta $367.5^{\circ}$ piso. Servicio de Medicina. Fono: 02-3543151 Fax: 02- 6394985

E-mail: asaizman@uc.cl

\section{Secondary adrenal insufficiency presenting as hyponatremia. Report of one case}

Hyponatremia can be a marker of an underlying disease. We report a 52 years-old male with Diabetes Mellitus who consulted for an episode of nausea and vomiting lasting four days. His baseline serum sodium was $118 \mathrm{mEq} / \mathrm{L}$. He had no neurological deficit. Hyponatremia was initially interpreted in context of gastrointestinal fluid loss but correction with saline solution was poor. His urine sodium was $105 \mathrm{mEq} / \mathrm{L}$ and his urine osmolality was $281 \mathrm{mOsm} / \mathrm{L}$, so an Inappropriate Secretion of Antidiuretic Hormone Syndrome was suspected. Later, we found that the patient had a two year history of fatigue, weakness, anorexia, frequent nausea, vomiting and diarrhea, loss of libido and decreased axillary and pubic hair. Thyroid-Stimulating Hormone (TSH) was normal and serum Cortisol $<1 \mu \mathrm{g} / d \mathrm{~L}$. A CT scan showed a sellar mass compatible with a macroadenoma. There was also a moderately high serum prolactin and low testosterone, thyroxin and growth hormone levels. The visual field examination showed right temporal hemianopsia. The patient was treated with steroids with a very good clinical response and serum sodium normalization. Subsequently a transsphenoidal excision of the tumor was performed and replacement of the other hormones was started. Now the patient remains asymptomatic.

(Rev Med Chile 2010; 138: 1144-1147).

Key words: Adrenal insufficiency; Hyponatremia; Pituitary neoplasms.
L a hiponatremia es la alteración electrolítica más frecuente en pacientes hospitalizados ${ }^{1}$. Sus principales causas son la contracción de volumen extracelular (VEC) por pérdidas gastrointestinales o renales y el síndrome de secreción inapropiada de hormona antidiurética (SIADH) ${ }^{2}$.

La anamnesis y el examen físico constituyen herramientas útiles al momento del enfrentamiento diagnóstico. Sin embargo, en ocasiones las alteraciones clínicas no permiten aclararlo y llegar a la etiología se transforma en un desafío para el tratante.

Comunicamos el caso de un hombre hospitalzado por hiponatremia severa atribuida inicialmente a disminución del VEC secundario a vómitos.

Durante su evolución, un examen sencillo como el sodio urinario permite reorientar la aproximación diagnóstica e identificar la patología de base.

\section{Caso clínico}

Hombre de 52 años, con antecedentes de diabetes mellitus tipo 2 de 6 años de evolución en tratamiento con dieta y retinopatía diabética. Consulta en el Servicio de Urgencia del Hospital Clínico de la Pontificia Universidad Católica de Chile el día 27 de febrero de 2009 por vómitos 
de cuatro días de evolución, sin otros síntomas asociados. Al examen físico destaca Presión arterial 114/64, frecuencia cardíaca 82 por minuto, mucosa oral y piel seca, abdomen normal, sin déficit neurológico. El laboratorio mostró sodio plasmático $118 \mathrm{mEq} / \mathrm{L}$, nitrógeno ureico $11 \mathrm{mg} / \mathrm{dl}$ (normal: $8-25$ ), creatininemia $0,67 \mathrm{mg} / \mathrm{dl}$ (normal: $0,5-0,9)$, potasio plasmático $4,3 \mathrm{mEq} / \mathrm{L}$ (normal: $3,5-5,0$ ), uricemia $3,4 \mathrm{mg} / \mathrm{dl}$ (normal: 3,6-8,5), hematocrito $32,5 \%$ con volumen corpuscular medio de $83,6 \mathrm{fl}$. Se decide hospitalización en la Unidad de Cuidados Intermedios.

La situación clínica se interpretó como una hiponatremia asociada VEC disminuido en contexto del síndrome emético. Se indicó aporte de solución salina al $9 \%$ a $80 \mathrm{ml} / \mathrm{h}$ (aprox 2 litros/día) con lo cual se logró un leve incremento de la natremia a $122 \mathrm{mEq} / \mathrm{L}$ en $24 \mathrm{~h}$. Frente a esta respuesta parcial y la presencia de nitrógeno ureico normal y uricemia disminuida, se midió sodio y osmolaridad urinaria cuyos resultados fueron $105 \mathrm{mEq} / \mathrm{L}$ y 281 $\mathrm{mOsm} / \mathrm{L}$ respectivamente. Dado la presencia de sodio y osmolaridad en orina elevados, se sospechó SIADH u otro mecanismo patogénico.

Se solicitaron niveles de tirotropina (TSH) y cortisol plasmático para descartar hipotiroidismo e insuficiencia suprarrenal. TSH: $3,17 \mu \mathrm{UI} / \mathrm{ml}$ (valor normal: $0,3-4,2 \mu \mathrm{UI} / \mathrm{ml}$ ) y cortisol: $<1 \mu \mathrm{g} /$

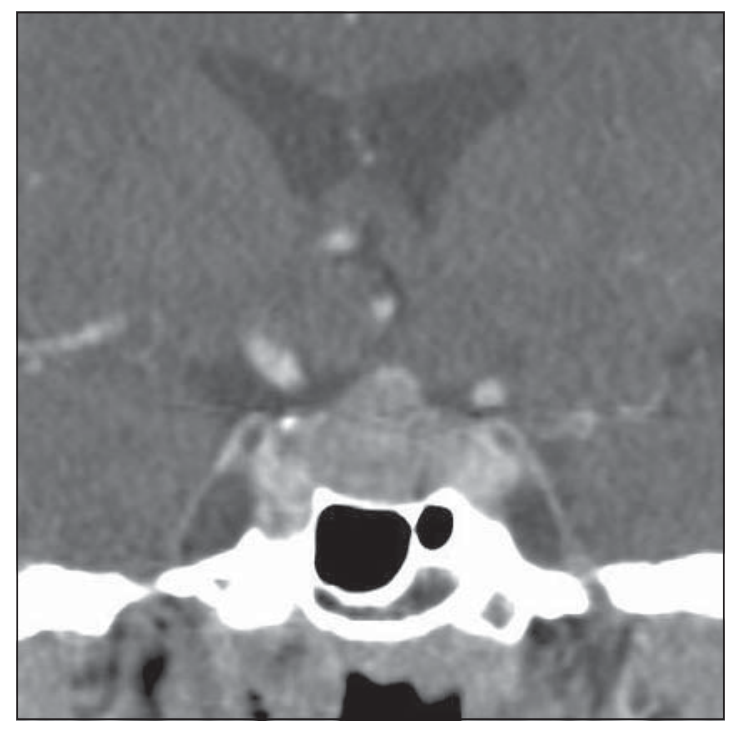

Figura 1. Tomografía computada encefálica que muestra tumor selar sugerente de adenoma hipofisiario.
dL (normal: 6,4-15 $\mu \mathrm{g} / \mathrm{dL}$ ). Se diagnosticó hiponatremia secundaria a hipocortisolismo.

Reinterrogando al paciente, refería historia de 2 a 3 años de fatigabilidad, debilidad y dolor muscular, astenia y adinamia. Presentaba también anorexia, episodios de náuseas, vómitos y diarrea intermitentes. Refería además disminución de la libido, pérdida del vello axilar y púbico. Respecto a su diabetes, presentaba un buen control de la glicemia exclusivamente con dieta (HbA1C 6,7\% en enero 2009).

Como parte de su estudio se decide realizar TAC encefálico que mostró masa selar sugerente de adenoma hipofisiario (Figura 1).

Fue evaluado por equipo de endocrinología quienes complementan estudio: prolactinemia: $50 \mathrm{ng} / \mathrm{ml}$ (normal: 2,1-20 ng/ml), somatostatina: $26 \mathrm{ng} / \mathrm{ml}$ (normal: 87-238 ng/ml), testosterona: 12 ng/dL (normal: 280-1100 ng/dL), T3: $74.7 \mathrm{ng} / \mathrm{dL}$ (normal: $80-200 \mathrm{ug} / \mathrm{dL}$ ) y T4: $5,5 \mathrm{mg} / \mathrm{dL}$ (normal: 4,6-12 $\mu \mathrm{g} / \mathrm{dL}$ ), revelando compromiso de todos los ejes hipotálamo-hipofisiarios. Además se efectúó RNM de silla turca que confirmó masa selar sugerente de adenoma de 14 x 16 x $17 \mathrm{~mm}$ con extensión supraselar, desplazamiento del quiasma óptico y desviación del tallo hipofisiario (Figura 2). Se evalúó campo visual mediante campimetría que revela hemianopsia temporal derecha.

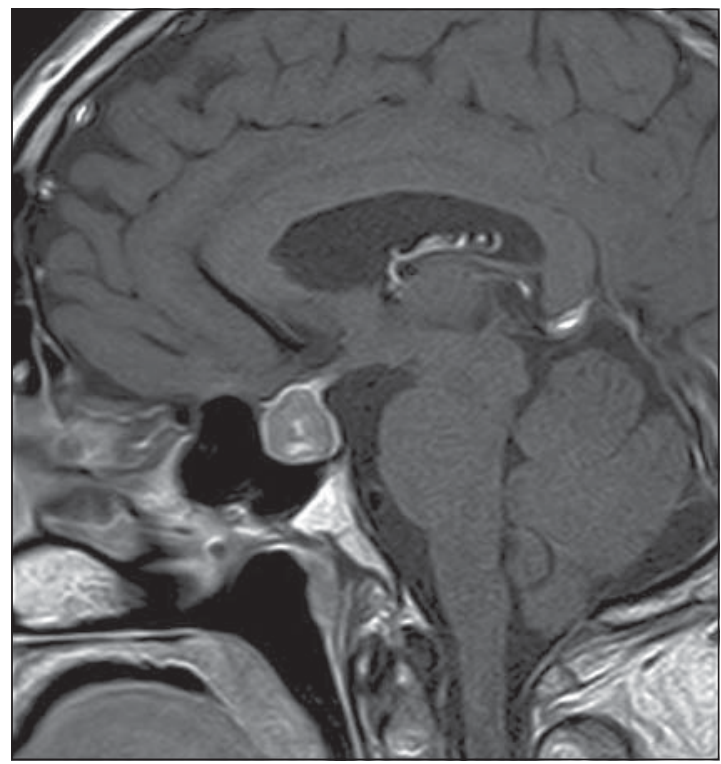

Figura 2. Resonancia Magnética de paciente que confirma masa selar sugerente de adenoma hipofisiario de 14 x $16 \times$ $17 \mathrm{~mm}$. 
Inició tratamiento con cortisol logrando natremia de $133 \mathrm{mEq} / \mathrm{L}$ y remisión gradual de sintomatología. Asimismo inició aporte de levotiroxina con $50 \mathrm{mcg} / \mathrm{d}$.

Se realizó resección transesfenoidal del adenoma el día 19 de marzo sin incidentes y posteriormente inició aporte de testosterona.

\section{Discusión}

La hiponatremia es una condición frecuente. En pacientes hospitalizados, 15 a 22\% tienen hiponatremia leve $e^{3}$ y a $7 \%$ hiponatremia moderada ${ }^{4-5}$. Sus causas pueden clasificarse en aquellas con osmolaridad plasmática normal-alta (pseudohiponatremia), y con osmolaridad baja. Estas últimas se subdividen en tres categorías:

1) Con VEC alto: ej: daño hepático crónico, insuficiencia cardíaca, síndrome nefrótico entre otros.

2) Con VEC normal: ej: SIADH, hipocortisolismo, hipotiroidismo y polidipsia primaria.

3) Con VEC bajo: ej: pérdidas gastrointestinales, tiazidas, hipoaldosteronismo y otros.

Las causas más frecuentes son aquellas con VEC disminuido y SIADH ${ }^{2}$.

Es importante señalar que la concentración plasmática de sodio refleja exactamente eso, concentración y no volumen. En otras palabras, la relación entre el sodio y el agua pero no la cantidad absoluta de ninguno de ellos. En el caso de las hiponatremias asociadas a pérdidas gastrointestinales (VEC bajo), los fluídos que se pierden suelen ser isosmóticos en relación al plasma. La hiponatremia se desarrolla entonces, como consecuencia de una reposición con fluídos hipotónicos (ingesta de agua) y con la activación de ADH mediada por estímulos hemodinámicas (no osmóticos). En pacientes con menor acceso al agua (lactantes y ancianos), estos cuadros suelen producir más bien hipernaremia.

Si bien en un principio se interpretó la hiponatremia como asociada a un VEC bajo en contexto del cuadro emético, existían varios elementos que hacían sospechar otras bases fisiopatológicas. El paciente, a pesar de tener una hiponatremia severa de $118 \mathrm{mEq} / \mathrm{L}$, no tenía compromiso de conciencia lo que sugería una evolución crónica. Por otro lado, llamaba la atención que un paciente diabético de años de evolución y retinopatía, tuviese buen control glicémico (HbAlc: 6,7\%) con dieta como único tratamiento.

A los argumentos anteriormente descritos se suma la presencia de nitrógeno ureico normal y uricemia bajo el valor normal (hallazgos no sugerentes de VEC bajo). Por esas razones se decide complementar el estudio midiendo el sodio urinario, cuyo resultado fue $105 \mathrm{mEq} / \mathrm{L}$. Esta cifra supone un volemia efectiva normal o elevada.

Se definen como criterios de SIADH: euvolemia clínica, osmolaridad plasmática menor a 275 $\mathrm{mOsm} / \mathrm{Kg}$, osmolaridad urinaria mayor a 100 $\mathrm{mOsm} / \mathrm{Kg}$, sodio urinario mayor a $40 \mathrm{mEq} / \mathrm{L}$, ausencia de uso de diuréticos y ausencia de hipotiroidismo e hipocortisolismo. La función cardíaca, hepática y renal también deben ser normales ${ }^{6}$. En nuestro caso, el cortisol plasmático disminuido permitió hacer el diagnóstico de hipocortisolismo pese a que se cumplían los otros criterios diagnósticos de SIADH. Esto constituye un pseudo SIADH. Es llamado de esta forma porque existe un SIADH producto del déficit de cortisol, el cual en concentraciones normales inhibe la liberación de vasopresina ${ }^{7}$.

La ausencia de hiperpigmentación e hiperkalemia, sumado a sintomatología sugerente de panhipopituitarismo orientaron a un hipercortisolismo secundario (compromiso hipofisiario). Es importante recordar también que la insuficiencia suprarrenal secundaria se asocia a hipoglicemia por compromiso concomitante de la hormona de crecimiento (en este caso, buen control glicémico sólo con dieta).

La causa más frecuente de panhipopituitarismo es un adenoma hipofisiario ${ }^{8}$.

Los adenomas de la hipófisis son tumores benignos originados en uno de los cinco tipos celulares de la hipófisis anterior. Representan alrededor del $10 \%$ de todas las neoplasias intracraneales $^{11}$. Estudios realizados con autopsias e imágenes reportan que en 10 a $20 \%$ de la población se encuentran microadenomas $(<10 \mathrm{~mm}$ de diámetro) asintomáticos. La tercera parte de todos los adenomas son no funcionantes 9 . Las formas de presentación más frecuentes son las derivadas del efecto de masa ${ }^{10-12}$ : hemianopsia bitemporal, disminución de la agudeza visual, cefalea, diplopia y pérdida nasal de líquido cefaloraquídeo. También se descubren como un hallazgo incidental en estudio de imágenes y por alteraciones hormonales. 
Dentro de éstas últimas, los estudios revelan que el déficit de la hormona del crecimiento es el más frecuente, luego el hipogonadismo, posteriormente hipotiroidismo y finalmente hipocortisolismo ${ }^{8}$.

Al momento del diagnóstico, es necesario continuar el estudio con la evaluación de los distintos ejes hipotálamo-hipofisiarios y la función neuroftalmológica. En el caso que se reporta, la prolactina de $50 \mathrm{ng} / \mathrm{ml}$ (levemente aumentada) se interpretó como secundaria a la compresión del tallo pituitario y no a un prolactinoma, ya que el valor no excedía los $100 \mathrm{ng} / \mathrm{ml}$, como suele ocurrir en éstos últimos ${ }^{10}$.

En resumen, la hiponatremia es un trastorno electrolítico frecuente, sobretodo en el ambiente intrahospitalario. Como hemos visto, es de mucha relevancia identificar la etiología que subyace a esta condición realizando una detallada anamnesis, examen físico y exámenes de laboratorio correspondientes. Es importante destacar que la impresión diagnóstica inicial en el caso analizado fue de una hiponatremia asociada a VEC bajo. Sin embargo, un examen tan sencillo como el sodio urinario permitió reorientar la aproximación diagnóstica en forma adecuada. De esta forma se logró llegar al diagnóstico de panhipopituitarismo, pudiendo iniciarse un tratamiento adecuado tanto médico como quirúrgico, mejorando considerablemente la calidad de vida del paciente.

\section{Referencias}

1. Adrogué HJ MD, Madias NE MD. Hyponatremia. N Engl J Med 2000; 342: 1581-9.

2. Pham PC, Pham PM, Pham PT. Vasopressin excess and hyponatremia. Am J Kidney Dis 2006; 47: 727-37.

3. Hawkins RC. Age and gender as risk factors for hyponatremia and hypernatremia. Clin Chim Acta 2003; 337: 169-72.

4. Anderson RJ, Chung H-M, Kluge R, Schrier RW. Hyponatremia: a prospective analysis of its epidemiology and the pathogenetic role of vasopressin. Ann Intern Med
1985; 102: 164-8.

5. Upadhyay A, Jaber BL, Madias NE. Incidence and prevalence of hyponatremia. Am J Med 2006; 119: Suppl 1: S30-5.

6. Ellison DH MD, Berl T MD. The Syndrome of Inappropiate Antidiuresis. N Engl J Med 2007; 356: 2064-72.

7. Oelkers W MD. Adrenal insuffiency. N Engl J Med 1996; 335: 1206-12.

8. López JM, Rodríguez JA. Endocrinología Clínica. En: Rodríguez JA, editores. Santiago, Chile. Editorial Mediterráneo; 2000. p. 50-2.

9. Rodríguez JA. Endocrinología Clínica. En: Rodríguez JA, editores. Santiago, Chile. Editorial Mediterráneo; 2000; p. 53-60.

10. Rodríguez JA. Endocrinología Clínica. En: Rodríguez JA, editores. Santiago, Chile. Editorial Mediterráneo; 2000; p. 61-4.

11. Gsponer J, De Tribolet N, Deruaz JP, Janzer R, Uske A, Mirimanoff RO, et al. Diagnosis, treatment, and outcome of pituitary tumors and other abnormal intrasellar masses. Retrospective analysis of 353 patients. Medicine (Baltimore) 1999; 78: 236-69.

12. Snyder PJ. Gonadotroph cell adenomas of the pituitary. Endocr Rev 19851; 6: 552-63.

13. Ciric I, Ragin A, Baumgartner C, Pierce D. Complications of transsphenoidal surgery: results of a national survey, review of the literature, and personal experience. Neosurgery 1997; 40: 225-36; discussion 236-7.

14. American Diabetes Association. Standards of medical care in diabetes 2006. Diabetes Care 2006; 29 Suppl 1: S4-42.

15. UKPDS 35. Association of Glycaemia with macrovascular and microvascular complications of tipe 2 Diabetes. BMJ 2000; 321: 405-12.

16. Stacpoole PW, Interlandi JW, Nicholson WE, Rabin D. Isolated ACTH deficiency: a heterogeneous disorder. Critical review and report of four new cases. Medicine (Baltimore) 1982; 61: 13-24.

17. Bates AS, Van'T Hoff W, Jones PJ, Clayton RN. The effect of hypopituitarism on life expectancy. J Clin Endocrinol Metab 1996; 81: 1169-72.

18. Molitch ME, Russell EJ. The pituitary "incidentaloma". Ann Intern Med 1990; 112: 925-31. 